\title{
The prognostic relevance of FOXA1 and Nestin expression in breast cancer metastases: a retrospective study of 164 cases during a 10-year period (2004-2014)
}

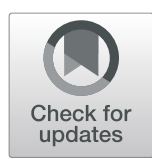

Shahin De Lara ${ }^{1 \dagger}$, Jenny Nyqvist ${ }^{2 \dagger}$, Elisabeth Werner Rönnerman ${ }^{1,3}$, Khalil Helou $^{3}$, Elisabeth Kenne Sarenmalm Zakaria Einbeigi ${ }^{5}$, Per Karlsson ${ }^{3,5}$, Toshima Z. Parris ${ }^{3+}$ and Anikó Kovács ${ }^{1 *+}$ (i)

\begin{abstract}
Background: Current prognostic markers cannot adequately predict the clinical outcome of breast cancer patients. Therefore, additional biomarkers need to be included in routine immune panels. FOXA1 was a significant predictor of favorable outcome in primary breast cancer, while Nestin expression is preferentially found in triple-negative tumors with increased rate of nodal metastases, and reduced survival. No studies have investigated the prognostic value of FOXA1 and Nestin expression in breast cancer metastases.

Methods: Breast cancer metastases $(n=164)$ from various anatomical sites were retrospectively analyzed by immunohistochemistry for FOXA1, Nestin and GATA3 expression. Cox regression analysis assessed the prognostic value of FOXA1 and Nestin expression.

Results: In breast cancer metastases, FOXA1 expression was associated with Nestin-negativity, GATA3-positivity, ER-positivity, HER2-positivity and non-triple-negative status $(P<0.05)$. In contrast, Nestin expression was associated with FOXA1-negative, GATA3-negative, ER-negative, and triple-negative metastases $(P<0.05)$. Univariate Cox regression analysis showed FOXA1 expression was predictive of overall survival $(O S, P=0.00048)$ and metastasis-free survival (DMFS, $P=0.0011)$, as well as, distant metastasis-free survival in ER-positive patients $(P=0.036)$ and overall survival in ER-negative patients $(P=0.024)$. Multivariate analysis confirmed the significance of FOXA1 for both survival endpoints in metastatic breast cancer patients (OS, $P=0.0033$; DMFS, $P=0.015$ ).

Conclusions: In our study, FOXA1 was expressed mostly in ER-positive breast cancer metastases. Expression of Nestin was related to triple-negative metastases, where brain was the most frequent metastatic site. These findings highlight the clinical utility of FOXA1 and Nestin expression and warrant their inclusion in routine immunohistochemical panels for breast carcinoma.
\end{abstract}

Keywords: FOXA1, Nestin, GATA3, Breast cancer metastases, Immunohistochemistry

\footnotetext{
* Correspondence: aniko.kovacs@vgregion.se

Shahin De Lara and Jenny Nyqvist contributed equally as first authors.

Toshima Z. Parris and Anikó Kovács contributed equally as last authors.

'Department of Clinical Pathology, Sahlgrenska University Hospital, Gula

stråket 8, SE-41345 Gothenburg, Sweden

Full list of author information is available at the end of the article
}

(c) The Author(s). 2019 Open Access This article is distributed under the terms of the Creative Commons Attribution 4.0 International License (http://creativecommons.org/licenses/by/4.0/), which permits unrestricted use, distribution, and reproduction in any medium, provided you give appropriate credit to the original author(s) and the source, provide a link to the Creative Commons license, and indicate if changes were made. The Creative Commons Public Domain Dedication waiver (http://creativecommons.org/publicdomain/zero/1.0/) applies to the data made available in this article, unless otherwise stated. 


\section{Background}

Immunohistochemical (IHC) markers for breast cancer (e.g. estrogen and progesterone receptors, Ki67 proliferation marker, and HER2/neu testing) are currently used to guide therapeutic decision-making, classify breast cancer subtypes, and act as prognostic and predictive markers $[1,2]$. However, these markers are still inadequate in certain subgroups of breast cancer patients. Nevertheless, great efforts have been made to identify novel molecular and IHC markers for clinical management of breast carcinoma that are informative, sensitive, and cost effective.

FOXA1 (forkhead box protein A1) is one of three members in the FOXA related family (forkhead family of transcriptions factors), also known as hepatocyte nuclear factor $\alpha(\mathrm{HNF} 3 \alpha)$. These proteins are often termed "pioneer factors" because of their ability to bind to highly compacted heterochromatin and making genomic regions more accessible to other transcription factors [3, 4]. Namely, FOXA1 can bind to the promotors of more than 100 genes associated with metabolic processes, regulation of the signaling pathways, and the cell cycle [4-6]. In addition, FOXA1 functions as a critical mediator of nuclear steroid receptor signaling via regulation of both androgen and estrogen receptor activity. FOXA1 is expressed in several organs, including the breast, liver, pancreas, urinary bladder, prostate gland, colon and lung. It has a major role in modulating nuclear steroid receptor activity, particularly in cancers of the breast and prostate, and may contribute to pro-tumorigenic phenotypes [7]. FOXA1 is a critical interacting partner of the nuclear hormone receptors, estrogen receptor $(E R \alpha)$ and androgen receptor (AR), which are associated with hormone-regulated cancers such as breast and prostate cancer $[3,8]$. FOXA1 is mutated in $1.8 \%$ of breast cancers and $3-5 \%$ of prostate cancers $[9,10]$. Nakshatri et al. reported that a decrease in FOXA1 expression during cancer progression was due to an increase in polycomb complex activity that plays a role in silencing Hox genes through modulation of chromatin structure during embryonic development [11]. The role of E-cadherin is established as an important player in epithelial-mesenchymal-transition (EMT). FOXA1 promotes E-cadherin expression on the protein level by suppressing Slug expression in breast cancer, suggesting that the balance of Foxa1-slug axis regulates EMT-progression [12]. FOXA1 positivity has also been linked with a more favorable prognosis in breast cancer patients treated with Tamoxifen [13, 14]. Reduction in FOXA1 expression contributes to cancer stem cell-like properties in Tamoxifen-resistant breast cancer cells through induction of Interleukin-6 (IL-6) [15]. Horimoto et al. reported that breast cancer patients with high FOXA1 expression tended to develop late recurrences [16].
There are currently few reports on the structure, function and clinical importance of Nestin in breast cancer. Nestin is a type VI intermediate filament protein encoded by the NES gene (identified originally as a neural stem cell marker) and participates in cytoskeleton organization [17]. It is expressed in proliferating progenitor cells in embryonic tissues, some adult stem/progenitor cells, and can even be re-expressed in neoplasia [18]. Triple-negative breast cancers have significantly higher NES (nestin) mRNA expression than the other breast carcinoma subtypes [19]. Nestin might participate in neovascularization through cytoskeletal changes promoted by the interaction between cancer cells with stem cell properties and endothelial cells lining blood vessels in the tumor stroma. Nowak et al. reported that Nestin expression in endothelial cells lining newly formed breast tumor-associated blood vessels was shown to be associated with the triple-negative subtype, lymph node metastases and shorter overall survival [17, 20]. Nestin contributes to activation of the EMT pathway by regulating the $\mathrm{Wnt} / \beta$-catenin pathway [21] and may therefore play a role not only in the regulation of mitosis, but in tumor invasiveness [18]. Piras et al. proposed Nestin-positivity in peritumoral stroma, in cells with fibroblast morphology, as evidence of epithelial-stromal interactions [22]. Nestin was significantly associated with angiogenesis and vascular invasion as a sign of early hematogenous spread, but not with lymphatic involvement [23]. In addition, Nestin-positive breast carcinomas lacked $C C N D 1$ and TOPA2A gene amplification and occasionally harbored $M Y C$ gene amplification. There was no correlation between Nestin expression and Topoisomerase II $\alpha$ expression [18]. Knockdown of Nestin inhibited breast cancer stem cell invasiveness and led to up-regulation of E-cadherin. Simultaneously, mesenchymal markers such as $\mathrm{N}$-cadherin and vimentin were down-regulated [19]. Feng et al. reported that enforced Nestin expression partly counteracted the effect of SOX10 knockdown on reducing cancer stem cell (CSC) properties.

In this present study, FOXA1 and Nestin expression were examined using immunohistochemistry for 164 breast cancer metastases, followed by Cox regression analysis to assess their prognostic significance in breast cancer.

\section{Methods}

\section{Patient cohort}

We examined 164 breast cancer metastases, corresponding to 162 patients diagnosed between a 10-year period 20042014 at Sahlgrenska University Hospital (Gothenburg, Sweden). Consequently, 2/164 patients were diagnosed with two metastases during this time period, including a 65-year-old patient with synchronous bone and brain metastases and a 29-year-old patient with 
Table 1 Clinicopathological features for 164 metastatic breast cancer patients

\begin{tabular}{|c|c|c|c|c|c|c|c|}
\hline & $\begin{array}{l}\text { Total patients } \\
(n=164)\end{array}$ & $\begin{array}{l}\text { FOXA1-positive } \\
(n=86)\end{array}$ & $\begin{array}{l}\text { FOXA1-negative } \\
(n=52)\end{array}$ & $P$-value & $\begin{array}{l}\text { Nestin-positive } \\
(n=26)\end{array}$ & $\begin{array}{l}\text { Nestin-negative } \\
(n=114)\end{array}$ & $P$-value \\
\hline Age (y) & & & & 0.21 & & & 0.29 \\
\hline$\leq 55$ & $59(36 \%)$ & $22(26 \%)$ & $20(38 \%)$ & & $10(38 \%)$ & $33(29 \%)$ & \\
\hline $56-80$ & $94(57 \%)$ & $59(69 \%)$ & $28(33 \%)$ & & $16(62 \%)$ & $72(63 \%)$ & \\
\hline$>80$ & $11(7 \%)$ & $5(6 \%)$ & $4(5 \%)$ & & $0(0 \%)$ & $9(8 \%)$ & \\
\hline Metastasic site & & & & 0.31 & & & 0.06 \\
\hline Abdomen/Gl tract/Liver & $44(27 \%)$ & $29(34 \%)$ & $9(17 \%)$ & & $5(19 \%)$ & $34(30 \%)$ & \\
\hline Axillary lymph node/sentinel node & $11(7 \%)$ & $5(6 \%)$ & $3(6 \%)$ & & $1(4 \%)$ & $7(6 \%)$ & \\
\hline Brain & $29(18 \%)$ & $7(8 \%)$ & $9(17 \%)$ & & $8(31 \%)$ & $9(8 \%)$ & \\
\hline Cervical lymph nodes & $3(2 \%)$ & $2(2 \%)$ & $1(2 \%)$ & & $0(0 \%)$ & $3(3 \%)$ & \\
\hline Gynecological site & $9(5 \%)$ & $2(2 \%)$ & $7(13 \%)$ & & $2(8 \%)$ & $7(6 \%)$ & \\
\hline Skeleton & $37(23 \%)$ & $20(23 \%)$ & 15 (29\%) & & $3(12 \%)$ & $32(28 \%)$ & \\
\hline Skin & $11(7 \%)$ & $7(8 \%)$ & $4(8 \%)$ & & $3(12 \%)$ & $8(7 \%)$ & \\
\hline Thorax/Lung & $20(12 \%)$ & $14(16 \%)$ & $4(8 \%)$ & & $4(15 \%)$ & $14(12 \%)$ & \\
\hline Not available & $0(0 \%)$ & $0(0 \%)$ & $0(0 \%)$ & & $0(0 \%)$ & $0(0 \%)$ & \\
\hline GATA3 status & & & & $2.80 \mathrm{E}-04$ & & & 0.039 \\
\hline Positive & 154 (94\%) & $86(100 \%)$ & $44(85 \%)$ & & $22(85 \%)$ & $110(96 \%)$ & \\
\hline Negative & $10(6 \%)$ & $0(0 \%)$ & $8(15 \%)$ & & $4(15 \%)$ & $4(4 \%)$ & \\
\hline Not available & $0(0 \%)$ & $0(0 \%)$ & $0(0 \%)$ & & $0(0 \%)$ & $0(0 \%)$ & \\
\hline Mammoglobin status & & & & 0.080 & & & 0.83 \\
\hline Positive & $84(51 \%)$ & $47(55 \%)$ & $20(38 \%)$ & & $12(46 \%)$ & $57(50 \%)$ & \\
\hline Negative & 80 (49\%) & $39(45 \%)$ & $32(62 \%)$ & & $14(54 \%)$ & $57(50 \%)$ & \\
\hline Not available & $0(0 \%)$ & $0(0 \%)$ & $0(0 \%)$ & & $0(0 \%)$ & $0(0 \%)$ & \\
\hline FOXA1 status & & & & & & & 0.0039 \\
\hline Positive & $86(52 \%)$ & - & - & & $9(35 \%)$ & 77 (68\%) & \\
\hline Negative & $52(32 \%)$ & - & - & & $17(65 \%)$ & $35(31 \%)$ & \\
\hline Not available & $26(16 \%)$ & - & - & & $0(0 \%)$ & $2(2 \%)$ & \\
\hline Nestin status & & & & 0.0017 & & & \\
\hline Positive & $26(16 \%)$ & $9(10 \%)$ & 17 (33\%) & & - & - & \\
\hline Negative & 114 (70\%) & 77 (90\%) & 35 (67\%) & & - & - & \\
\hline Not available & $24(15 \%)$ & $0(0 \%)$ & $0(0 \%)$ & & - & - & \\
\hline Primary tumor differentiation & & & & 0.29 & & & 0.96 \\
\hline Well & $10(6 \%)$ & $5(6 \%)$ & $3(6 \%)$ & & $1(4 \%)$ & $7(6 \%)$ & \\
\hline Moderate & $49(30 \%)$ & $31(36 \%)$ & $11(21 \%)$ & & $7(27 \%)$ & $36(32 \%)$ & \\
\hline Poor & $59(36 \%)$ & $27(31 \%)$ & 19 (37\%) & & $9(35 \%)$ & $37(32 \%)$ & \\
\hline Not available & $46(28 \%)$ & $23(27 \%)$ & 19 (37\%) & & $9(35 \%)$ & $34(30 \%)$ & \\
\hline Axillary lymph node status & & & & 0.047 & & & 1.00 \\
\hline pNO & $55(34 \%)$ & $34(40 \%)$ & $11(21 \%)$ & & $9(35 \%)$ & $37(32 \%)$ & \\
\hline pN1 & $78(48 \%)$ & $38(44 \%)$ & $26(50 \%)$ & & $12(46 \%)$ & $53(46 \%)$ & \\
\hline Not available & $31(19 \%)$ & $14(16 \%)$ & 15 (29\%) & & $5(19 \%)$ & $24(21 \%)$ & \\
\hline ER status & & & & 2.09E-05 & & & 0.024 \\
\hline Positive & $115(70 \%)$ & $74(86 \%)$ & $27(52 \%)$ & & $14(54 \%)$ & $89(78 \%)$ & \\
\hline Negative & 49 (30\%) & $12(14 \%)$ & $25(48 \%)$ & & $12(46 \%)$ & $25(22 \%)$ & \\
\hline Not available & $0(0 \%)$ & $0(0 \%)$ & $0(0 \%)$ & & $0(0 \%)$ & $0(0 \%)$ & \\
\hline
\end{tabular}


Table 1 Clinicopathological features for 164 metastatic breast cancer patients (Continued)

\begin{tabular}{|c|c|c|c|c|c|c|c|}
\hline & $\begin{array}{l}\text { Total patients } \\
(n=164)\end{array}$ & $\begin{array}{l}\text { FOXA1-positive } \\
(n=86)\end{array}$ & $\begin{array}{l}\text { FOXA1-negative } \\
(n=52)\end{array}$ & $P$-value & $\begin{array}{l}\text { Nestin-positive } \\
(n=26)\end{array}$ & $\begin{array}{l}\text { Nestin-negative } \\
(n=114)\end{array}$ & $P$-value \\
\hline PR status & & & & 0.07 & & & 0.50 \\
\hline Positive & $57(35 \%)$ & $39(45 \%)$ & $15(29 \%)$ & & $8(31 \%)$ & $46(40 \%)$ & \\
\hline Negative & $107(65 \%)$ & 47 (55\%) & 37 (71\%) & & $18(69 \%)$ & $68(60 \%)$ & \\
\hline Not available & $0(0 \%)$ & $0(0 \%)$ & $0(0 \%)$ & & $0(0 \%)$ & $0(0 \%)$ & \\
\hline HER2/neu status & & & & 0.0031 & & & 0.42 \\
\hline Positive & $45(27 \%)$ & $29(34 \%)$ & $6(12 \%)$ & & $4(15 \%)$ & $31(27 \%)$ & \\
\hline Negative & $116(71 \%)$ & $55(64 \%)$ & $46(88 \%)$ & & $22(85 \%)$ & $81(71 \%)$ & \\
\hline Not available & $3(2 \%)$ & $2(2 \%)$ & $0(0 \%)$ & & $0(0 \%)$ & $2(2 \%)$ & \\
\hline Triple negative status & & & & $1.92 \mathrm{E}-06$ & & & 0.0021 \\
\hline Triple negative & $27(16 \%)$ & $4(5 \%)$ & $19(37 \%)$ & & $10(38 \%)$ & $13(11 \%)$ & \\
\hline Non-triple negative & 137 (84\%) & $82(95 \%)$ & $33(63 \%)$ & & $16(62 \%)$ & $101(89 \%)$ & \\
\hline Not available & $0(0 \%)$ & $0(0 \%)$ & $0(0 \%)$ & & $0(0 \%)$ & $0(0 \%)$ & \\
\hline Overall survival & & & & 0.17 & & & 1.00 \\
\hline Deceased & $88(54 \%)$ & $68(79 \%)$ & $46(88 \%)$ & & $22(85 \%)$ & $94(82 \%)$ & \\
\hline Survivor & 76 (46\%) & 18 (21\%) & $6(12 \%)$ & & $4(15 \%)$ & 20 (18\%) & \\
\hline
\end{tabular}

NOTE: $P$-values were calculated using the Fisher's exact test (FOXA1-positive vs. FOXA1-negative or Nestin-positive vs Nestin-negative. Statistically significant variables $(p<0.05)$ are displayed in bold text

metachronous recurrent brain metastases with a 6-month interval. Nine of the 164 breast cancer metastases were regional axillary lymph node metastases (5.5\%), while 155 metastases were distantly located as indicated in Table 1.

In total, $20 / 162$ patients $(12.3 \%)$ were still alive at the start of the study (henceforth termed long-term survivors). For long-term survivors, the interval between the primary breast cancer and metastases varied between 2 and 35 years (average 8.25 years). However, three of the 20 patients developed metastases within 12 months. The youngest long-term survivor was 44-years-old at the time of metastasis, while the oldest patient was 82-years-old (average age was 58.6 years).

In addition, 11 of the 162 patients $(6.8 \%)$ had already been diagnosed with metastatic breast cancer at the time of diagnosis of the primary tumor. Of these, 3/11 patients had already developed axillary lymph node metastases at the time of diagnosis, 3/11 patients had bone metastases, 1/11 patient had a liver metastasis, 2/ 11 patients had metastases in the abdomen and peritoneum, 1/11 patient was diagnosed with breast cancer metastases in the ovarium, and 1/11 patient presented with breast cancer metastases in the soft tissue in the parasternal region.

\section{Evaluation of immunohistochemistry (IHC)}

Full-face formalin-fixed paraffin-embedded (FFPE) specimens for the 164 breast cancer metastases were obtained from the Department of Clinical Pathology at Sahlgrenska University Hospital in accordance with the Declaration of Helsinki and approved by the Medical Faculty Research Ethics Committee (Gothenburg, Sweden). Each FFPE specimen was examined for mammaglobin, ER/PR, cytokeratin 7 (CK7), cytokeratin 20 (CK20), and HercepTest at the time of diagnosis and retrospectively analyzed by immunohistochemistry (IHC) for GATA3, FOXA1 and Nestin expression. Four micrometer FFPE (formalin fixed paraffin embedded) sections were pretreated using the Dako PTLink system (Dako, Carpinteria, CA) and processed on an automated DAKO Autostainer platform using the Dako Envision ${ }^{\mathrm{Tm}}$ FLEX High $\mathrm{pH}$ Link Kit (pH 9; Table 2). Peroxidase-catalyzed diaminobenzidine was used as the chromogen, followed by hematoxylin counterstain. Normal breast gland tissue and tonsillar tissue were used as positive controls for FOXA1 and Nestin, respectively. A Leica DM4000 B microscope was used in the experiments. Immunostaining was evaluated by a breast pathologist, blinded

Table 2 Summary of immunohistochemical stains and antigen retrieval techniques

\begin{tabular}{|c|c|c|c|c|}
\hline Antibody & Manufacturer & Clone & Dilution & Antigen retrieval \\
\hline$\overline{\text { GATA3 }}$ & $\begin{array}{l}\text { CELL MARQUE } \\
390 \mathrm{M}-16\end{array}$ & L50-823 & $1: 200$ & TRS high, pH 9.0 \\
\hline FOXA1 & $\begin{array}{l}\text { CELL MARQUE } \\
405 \mathrm{M}-16\end{array}$ & $2 F 83$ & 1:100 & TRS high, pH 9.0 \\
\hline Nestin & $\begin{array}{l}\text { CELL MARQUE } \\
388 \mathrm{M}-16\end{array}$ & $10 C 2$ & $1: 50$ & TRS high, pH 9.0 \\
\hline
\end{tabular}


to patient clinical outcome. For each metastasis, the pattern of nuclear FOXA1, nuclear GATA3, and cytoplasmic Nestin staining was recorded together with the extent (\% of positively stained tumor cell nuclei or tumor cell cytoplasm using deciles: $0 \% ; 5,10,40$, $90 \%$, etc.). Positivity thresholds were set at $\geq 1 \%$. However, $<20 \%$ was rarely noticed among positive cases. An overall assessment of IHC was given, not focusing on hot spots.

\section{Statistical analysis}

The statistical analyses were performed using a 0.05 $P$-value cutoff in R/Bioconductor (version 3.3.2) and all $P$-values are two-sided. The relationship between clinicopathological features and FOXA1 and Nestin protein expression patterns was evaluated using two-tailed Fisher's exact test ( $\mathrm{R}$ stats package). Univariate Cox proportional hazard models were calculated for FOXA1 and Nestin expression using overall survival (OS) and distant metastasis-free survival (DMFS; survival (v2.43-1)). Multivariate analysis was conducted using the Cox proportional hazard model for OS and DMFS with FOXA1 and Nestin expression after adjusting for clinicopathological features (age at diagnosis, metastatic site, Mammoglobin status, GATA3 status, histological grade, axillary lymph node status, ER/PgR status, HER2/neu status, and triple-negative status). Breast cancer survival rates were defined as a) time from initial diagnosis of the primary breast carcinoma to death from any cause for OS and b) time from initial diagnosis of the primary breast carcinoma to distant metastasis for DMFS. Survival rates were depicted
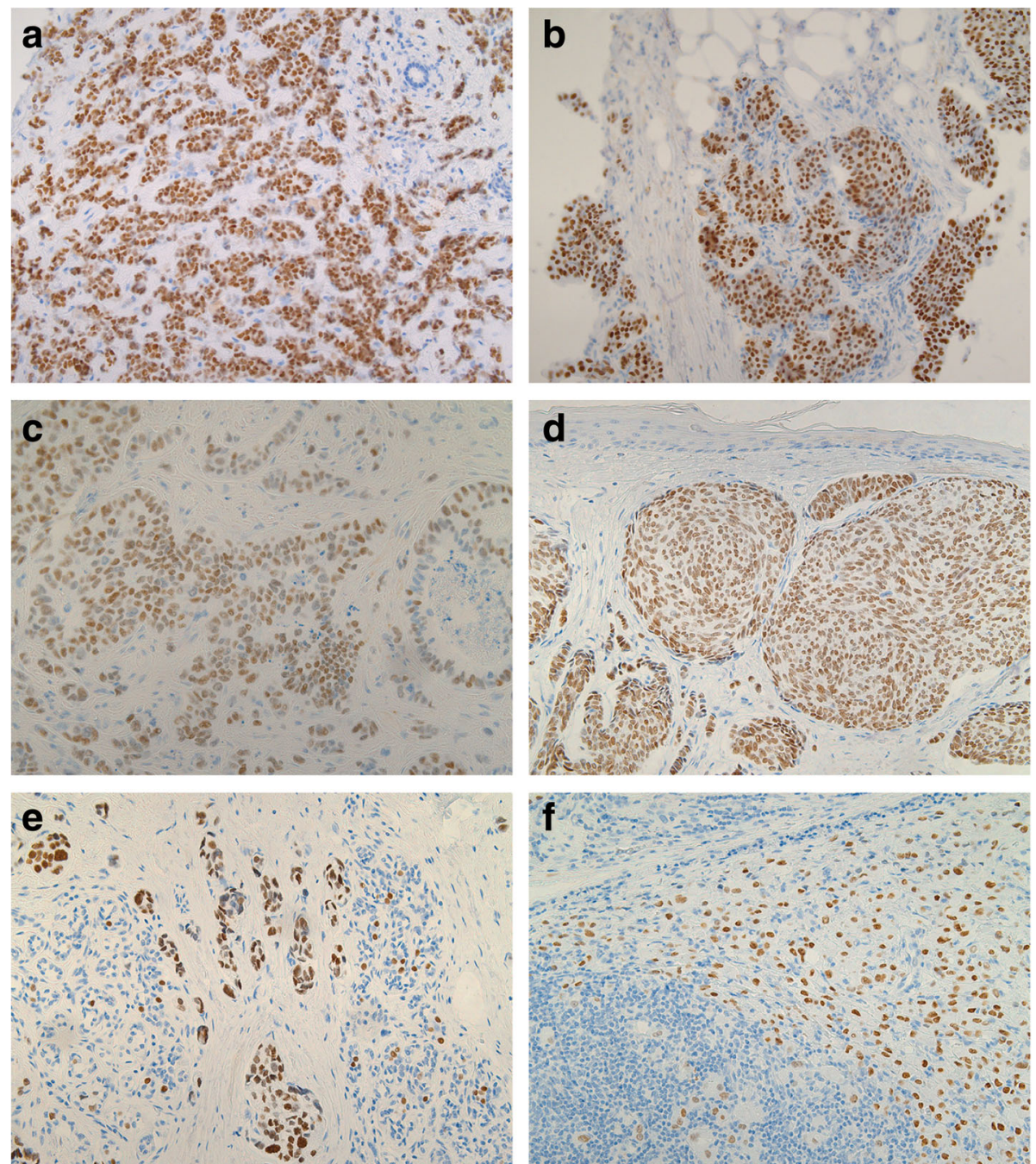

Fig. 1 Positive FOXA1 immunostaining in breast cancer metastases, including a liver, b mediastinum, c cerebellum, d skin, e breast with recurrent cancer, and $\mathbf{f}$ axillary lymph node (100 x magnification each) 
with Kaplan-Meier curves and tested with log-rank test (survminer (v0.4.3)). Forest plots were generated using the forestplot package (v1.7.2).

\section{Results}

\section{Association between clinicopathological features and} FOXA1 and nestin expression

Immunohistochemical analysis of FOXA1 and Nestin expression in breast cancer metastases revealed FOXA1positivity and Nestin-positivity in 52 and $16 \%$ of the metastatic samples, respectively (Fig. 1, 2 and Table 1). A strong association between FOXA1 expression and GATA3-positivity $(P<0.001)$ was found, with FOXA1 expression detected in $100 \%$ of GATA3-positive cases. In addition, FOXA1 expression was found in patients with no spread to the axillary lymph nodes and tumors characterized as Nestin-negative, ER-positive, HER2-positive, and non triple-negative $(P<0.05)$. In contrast, Nestin-positivity was inversely associated with GATA3, FOXA1, and ER expression, and triple negative status $(P<0.05)$. Hence, protein expression for FOXA1 and Nestin were inversely related. No association was found between FOXA1 and Nestin expression and other clinicopathological features, such as metastatic site, age, mammoglobin status, primary tumor differentiation, axillary lymph node status, progesterone receptor status or overall survival.

\section{Prognostic significance of FOXA1 and nestin expression in breast cancer metastases}

Univariate Cox regression analysis showed an association between FOXA1 expression and both overall survival (OS; $P=0.00048$ ) and distant metastasis-free survival (DMFS; $P=0.0011$; Fig. 3). In contrast, Nestin expression was not associated with clinical outcome in the patient cohort (OS and DMFS; $P>0.05$ ). Stratification of the patient cohort by ER status revealed that FOXA1 expression was a predictor of DMFS in ER-positive patients $(P=0.036)$ and OS in ER-negative patients $(P=0.024)$. No significant correlation was found between Nestin expression and either survival endpoint in patients stratified by ER status (Fig. 4, 5). Multivariate analysis using a Cox proportional hazards model was performed to assess the prognostic value of FOXA1 and Nestin expression after adjusting for other clinicopathological features. Interestingly, multivariate analysis confirmed the clinical relevance of FOXA1 expression and survival in metastatic breast cancer patients (OS, $P=0.010$; DMFS, $P=0.069$; Fig. 6). In addition, metastatic site (i.e. brain, cervical lymph nodes, GI tract, gynecological, liver, skeleton, skin, thorax), mammoglobin-positivity, and GATA3-positivity were associated with OS $(P<0.05)$. For DMFS, metastatic site (i.e. brain, cervical lymph nodes, liver, skeleton, skin,
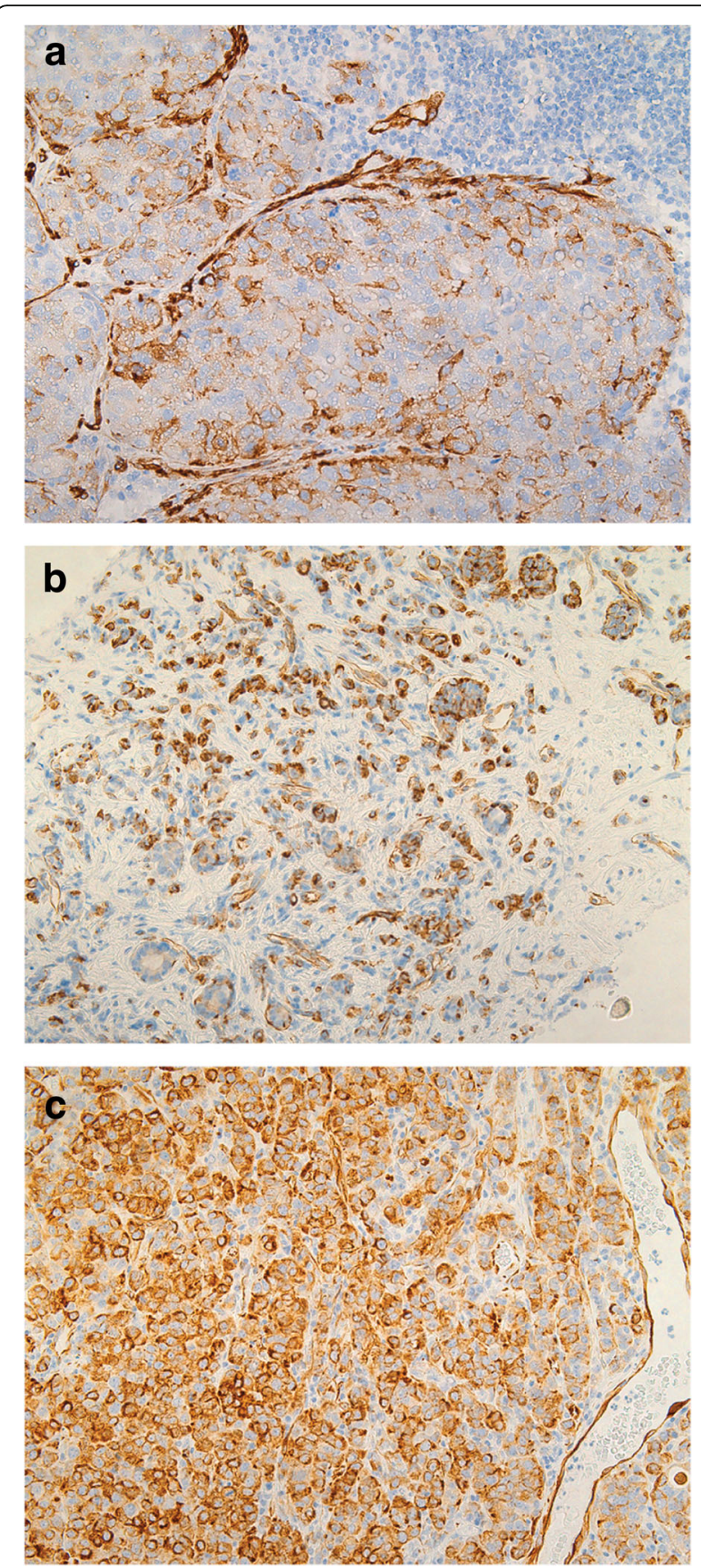

Fig. 2 Positive Nestin immunostaining in breast cancer metastases, including a sentinel node (200 x magnification), b liver "(100 x magnification)", and c cerebellum (200 x magnification)

thorax), mammoglobin-positivity, GATA3-positivity, HER2-positivity, triple-negative status, and high histological grade were statistically significant $(P<0.05)$.

\section{Discussion}

To date, immunohistochemical analysis of FOXA1 and Nestin expression has only been performed using 

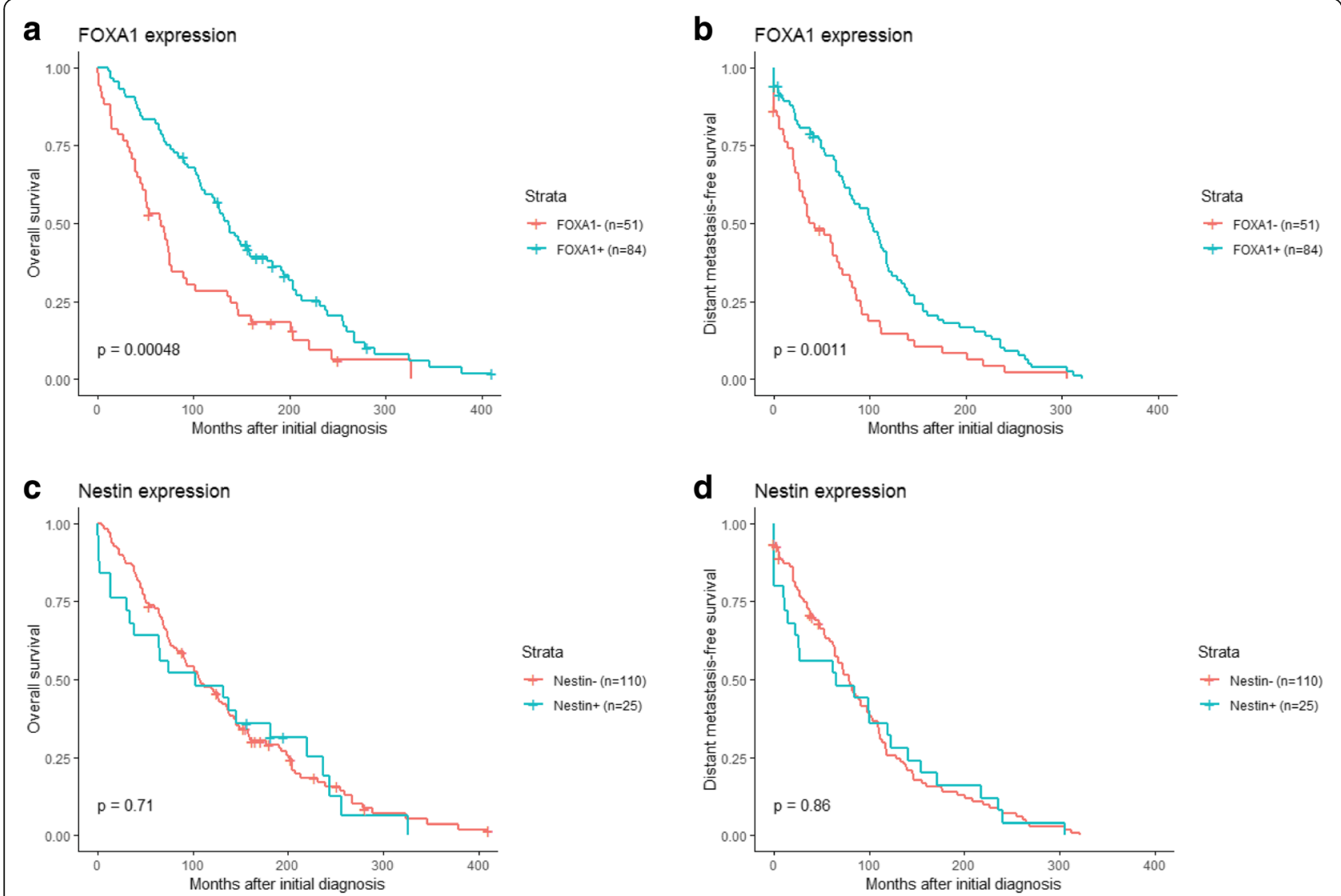

Fig. 3 Kaplan-Meier analysis of FOXA1 and Nestin expression in metastatic breast cancer patients. a and $\mathbf{c}$ Estimates of the probability of overall survival (OS) in the patient cohort. P-values were calculated using the log-rank test and Cox proportional hazards regression. $\mathbf{b}$ and $\mathbf{d}$ Estimates of the probability of distant metastasis-free survival (DMFS) in the patient cohort. $P$-values were calculated using the log-rank test and Cox proportional hazards regression. The $\mathrm{x}$-axes depict months after initial diagnosis and the $y$-axes depict survival rates

primary breast carcinomas. In the present study, only the breast cancer metastases were available, all of which had been diagnosed at Sahlgrenska University Hospital (Gothenburg, Sweden). Unfortunately, the FFPE samples for the primary breast carcinomas were more difficult to retrieve and the patients had been diagnosed and operated for the primary tumors at different hospitals in Western Sweden. It was therefore not possible to evaluate FOXA1 and Nestin expression in the primary tumor at this time. In future studies, it would be interesting to investigate FOXA1 and Nestin expression in the primary breast carcinoma corresponding to the 164 metastatic breast carcinomas investigated here. This is the first report, to our knowledge, showing FOXA1 and Nestin expression in breast cancer metastases.

Previous studies have shown that FOXA1 expression was inversely associated with tumor size, histological grade, lymph node status, HER2 expression and lymph vascular invasion, while GATA3 expression showed an inversed association with tumor histological grade and HER2 status [24, 25]. Both FOXA1 and GATA3 were associated with ER $\alpha$ and progesterone positivity. Over
$80 \%$ of FOXA1 and GATA3-positive breast carcinomas belonged to the luminal A subtype. FOXA1 have been shown to have a dual role, either as a growth stimulator or a repressor. It functions as a tumor promotor in initial stages, but as a tumor repressor in the later stages $[4,5]$. Cross talk between FOXA1 and ER can favor the expression of differentiation-associated genes, and not the proliferation-associated genes that results in well differentiated breast carcinomas with estrogen receptor positivity, which per se indicates a good prognosis [5] [26]. As a tumor suppressor, FOXA1 overexpression might block metastatic progression by influencing the expression of $p 27$ (BRCA1 associated cell cycle inhibitor) and promoting E-Cadherin expression [4].

According to the statistical analysis, our results correlated well with the data reported in the literature. Breast cancer patients with tumors expressing FOXA1 may have a better clinical outcome because FOXA1 and GATA3 are expressed in close association with ER $\alpha$ (Estrogen receptor $\alpha$ ), by encoding for transcription factors and have a potential involvement in ER $\alpha$-mediated breast cancer development [24]. Because FOXA1 was 

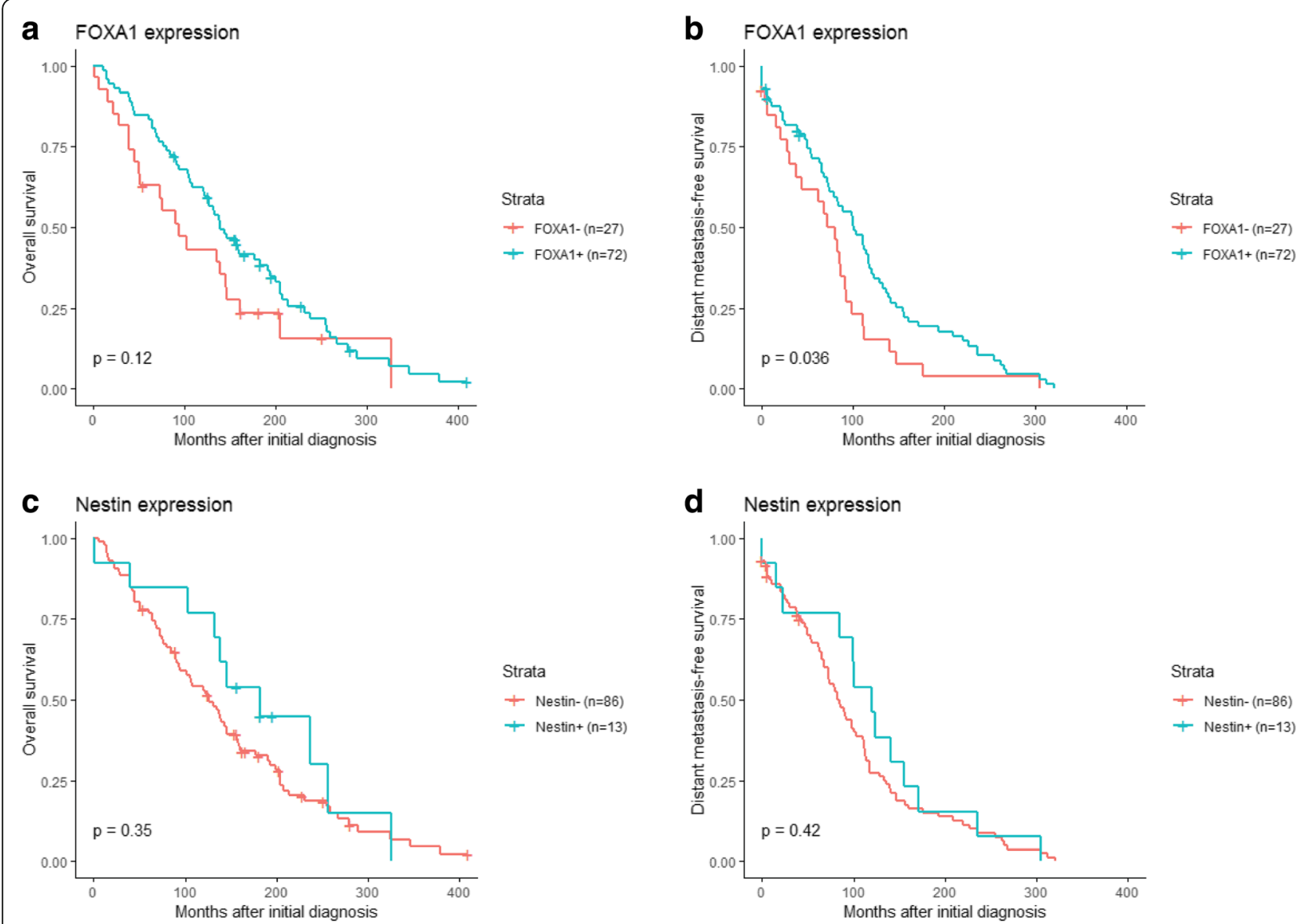

Fig. 4 Kaplan-Meier analysis of FOXA1 and Nestin expression in ER-positive, metastatic breast cancer patients. a and $\mathbf{c}$ Estimates of the probability of overall survival (OS) in the patient cohort. P-values were calculated using the log-rank test and Cox proportional hazards regression. $\mathbf{b}$ and $\mathbf{d}$ Estimates of the probability of distant metastasis-free survival (DMFS) in the patient cohort. $P$-values were calculated using the log-rank test and Cox proportional hazards regression. The x-axes depict months after initial diagnosis and the $y$-axes depict survival rates

clinically relevant in several studies, its practical application in routine pathology might be advisable. Ademuyiwa et al. suggested that FOXA1 immunostaining could function as a more cost effective pathological marker than the Oncotype DX multigene prognosis assay, because FOXA1 negatively correlated with the recurrence score [27]. FOXA1 is already included in PAM50, which is also a gene profiling kit for breast cancer patients. A decade has passed since Thorat et al. suggested FOXA1 be used in treatment decision making, as it showed a prognostic ability in low-risk breast cancer [26]. Moreover, neoadjuvant chemotherapy (NAC) could be recommended for breast cancer patients with low FOXA1 expression [16]. FOXA1 expression was also been evaluated after NAC, which was significantly associated with distant disease-free survival, both by univariate and multivariate analyses [28].

FOXA1 expression has also been assessed in primary carcinomas from other anatomic sites than breast carcinoma. Interestingly, FOXA1 DNA amplification was only observed in metastatic prostate cancer samples, which was associated with increased proliferation and tumor size [10, 29]. In breast cancer, FOXA1 amplification was a marker of favorable prognosis [3, 13]. Molecular classification of breast cancer is a helpful tool with respect to patient clinical outcome. Robinson et al. reported that the majority of breast cancers expressing FOXA1 were mainly of Luminal A type (5/8), Luminal B type (2/8), and HER2 positive subtype (1/8). Robinson et al. also reported that breast cancer patients with FOXA1 amplification have a much better prognosis and treatment response [3]. Decreased FOXA1 levels in pleural metastases was associated with endocrine therapy resistance [30].

While FOXA1 and GATA3 are significant predictors of favorable outcome in breast carcinoma (longer disease-free interval and overall survival), Nestin expression is associated with a more unfavorable prognosis [13, 24, 31]. Our results correlated well with these findings, as FOXA1 expression was associated with both 
a FOXA1 expression

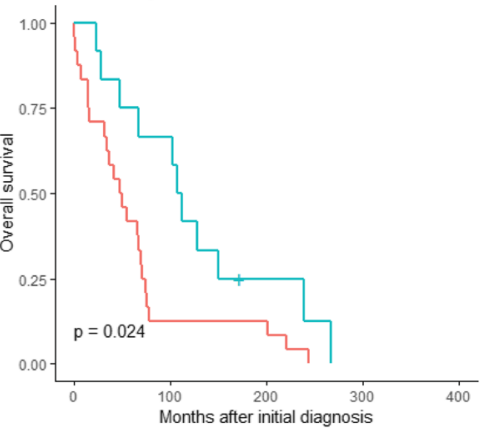

C Nestin expression

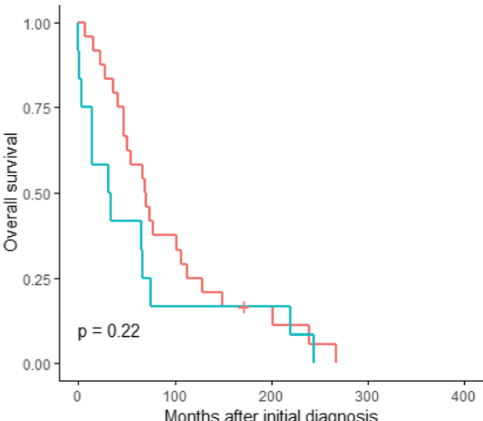

b FOXA1 expression

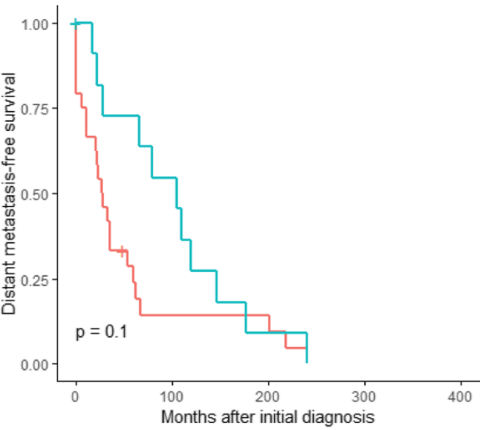

Strata

+ FOXA1-(n=24)

+ FoxA1+(n=12)

+ FOXA1- $(\mathrm{n}=24)$

+ FOXA1+ $(n=12)$

Strata

+ Nestin- (n=24)

+ Nestin+ $(n=12)$

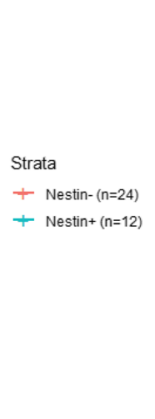

d Nestin expression

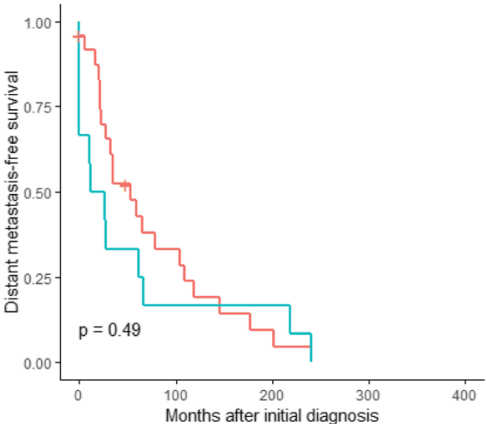

Strata

+ Nestin+ $(n=12)$
+ Nestin- (n=24)

Fig. 5 Kaplan-Meier analysis of FOXA1 and Nestin expression in ER-negative, metastatic breast cancer patients. a and $\mathbf{c}$ Estimates of the probability of overall survival (OS) in the patient cohort. $P$-values were calculated using the log-rank test and Cox proportional hazards regression. $\mathbf{b}$ and $\mathbf{d}$ Estimates of the probability of distant metastasis-free survival (DMFS) in the patient cohort. P-values were calculated using the log-rank test and Cox proportional hazards regression. The $\mathrm{x}$-axes depict months after initial diagnosis and the $\mathrm{y}$-axes depict survival rates

\section{a}

variables

Age

FOXA1, positive

Nestin, positive

ER, positive

PgR, positive

HER2, positive

Triple-negative

Mammoglobin, positive

GATA3, positive

Axillary Iymph node, positive

Metastatic site

Axillary lymph nodes

Brain

Cervical lymph nodes

G| tract

Gynecological

Liver

Skeleton

Skin

Thorax

Histological tumor grade

Poorly differentiated

Well-differentiated
Multivariate analysis: $0 S(n=135)$

Hazard ratio $(95 \% \mathrm{Cl}) \quad$ P-value

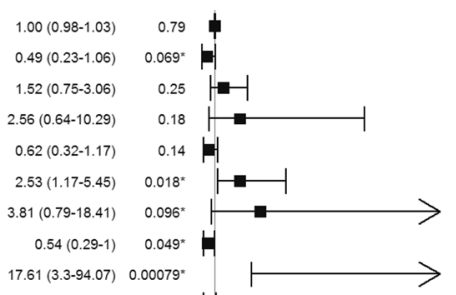

$0.58(0.3-1.11)$

0.1

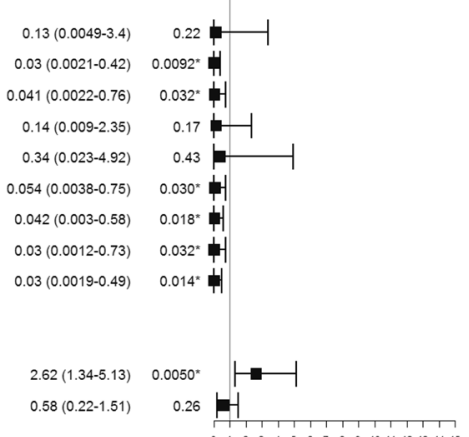

\section{b}

variables

Age

FOXA1, positive

Nestin, positive

ER, positive

PgR, positive

HER2, positive

Triple-negative

Mammoglobin, positive

GATA3, positive

Axillary Iymph node, positive

Metastatic site

Axillary lymph nodes

Brain

Cervical Iymph nodes

GI tract

Gynecological

Liver

Skeleton

Skin

Thorax

Histological tumor grade

Poorly differentiated

Well-differentiated
Multivariate analysis: DMFS $(n=135)$

Hazard ratio $(95 \% \mathrm{Cl}) \quad$ P-value

Fig. 6 Forest plots illustrating multivariate Cox regression analysis of the prognostic impact of FOXA1 and Nestin expression on, a overall survival (OS), and $\mathbf{b}$ distant metastasis-free survival (DMFS) in metastatic breast cancer patients 
better OS and DMFS. According to our data, Nestin was not associated with OS and DMFS, but was correlated with triple-negative status which is generally connected with poor patient outcome. Moreover, Nestin might become a potential treatment target because knockdown of Nestin demonstrated reduced cell motility in several different carcinomas, including prostate, colorectal-, nasopharyngeal- and lung carcinoma [23]. Here, we show FOXA1 expression in breast cancer metastases, but it is still unclear whether other FOXA family members such as FOXA2 and FOXA3 also play a role in breast cancer.

\section{Conclusions}

In the present study, FOXA1 and Nestin expression in breast cancer metastases was associated with specific breast cancer subtypes (luminal phenotype versus triple-negative breast cancer metastases). GATA3 has been already used as a specific marker to identify breast cancer metastases [32]. We propose the inclusion of FOXA1 and Nestin immunohistochemical evaluation in current immune panels for breast carcinoma to improve prognostication and therapy choice.

\section{Abbreviations \\ AR: androgen receptor; CK 20: cytokeratin 20; CK 7: cytokeratin 7; CSC: cancer stem cell; DMFS: distant metastasis-free survival; EMT: epithelial- mesenchymal-transition; ER: estrogen receptor; ERa: estrogen receptor a; FFPE: full-face formalin-fixed paraffin-embedded; FOXA1: forkhead box protein A1; GI-tract: gastrointestinal tract; HNF3a: hepatocyte nuclear factor a; IHC: immunohistochemistry; NAC: neoadjuvant chemotherapy; OS: overall survival}

\section{Acknowledgements}

We thank AH Diagnostics (Stockholm, Sweden) for generously providing the FOXA1 and Nestin antibodies.

\section{Funding}

Not applicable.

\section{Availability of data and materials}

The datasets used and analyzed during the current study are available from the corresponding author on reasonable request.

\section{Authors' contributions}

AK and JN were responsible for overall study concept. AK, JN, SDL, TZP were responsible for the design of experiments. AK, JN, ZE, PK, and EWR collected the clinical data. AK, JN, TZP contributed to the statistical analyses. SDL, AK, EKS, and KH provided technical and material support. AK, JN, and SDL performed the experiments. AK, JN, TZP analyzed the data, performed the statistical analyses, and wrote the manuscript. All authors reviewed, edited, and approved the final manuscript.

\section{Authors' information}

SDL and JN share first author status. AK and TZP share last author status.

\section{Ethics approval and consent to participate}

The study was ethically approved by the Sahlgrenska Academy Medical Faculty Research Committee, Gothenburg, Sweden (768-14). The requirement for informed consent was waived by the ethical committee since the patient material was stripped from direct subject identifiers. Prior to surgery, all patients have approved storage of material in the local medical biobank for clinical and research purposes.
Consent for publication

Not applicable.

\section{Competing interests}

The authors declare that they have no competing interests.

\section{Publisher's Note}

Springer Nature remains neutral with regard to jurisdictional claims in published maps and institutional affiliations.

\section{Author details}

'Department of Clinical Pathology, Sahlgrenska University Hospital, Gula stråket 8, SE-41345 Gothenburg, Sweden. ${ }^{2}$ Department of Surgery,

Skaraborgs Hospital, Lidköping and Sahlgrenska Academy at University of Gothenburg, Gothenburg, Sweden. ${ }^{3}$ Department of Oncology, Institute of Clinical Sciences, Sahlgrenska Cancer Center, Sahlgrenska Academy at University of Gothenburg, Gothenburg, Sweden. ${ }^{4}$ Center for Research and Development, Skaraborgs Hospital, Skövde, Sweden. ${ }^{5}$ Department of Oncology, Sahlgrenska University Hospital, Gothenburg, Sweden.

Received: 28 September 2018 Accepted: 17 February 2019

Published online: 28 February 2019

\section{References}

1. Gnant M, Harbeck N, St TC. Gallen/Vienna 2017: a brief summary of the consensus discussion about escalation and De-escalation of primary breast Cancer treatment. Breast care (Basel, Switzerland). 2017;12(2):102-7.

2. Morigi C. Highlights from the 15th St Gallen international breast Cancer conference 15-18 march, 2017, Vienna: tailored treatments for patients with early breast cancer. Ecancermedicalscience. 2017;11:732.

3. Robinson JL, Holmes KA, Carroll JS. FOXA1 mutations in hormonedependent cancers. Front Oncol. 2013;3:20.

4. Habashy HO, Powe DG, Rakha EA, Ball G, Paish C, Gee J, et al. Forkhead-box A1 (FOXA1) expression in breast cancer and its prognostic significance. European journal of cancer (Oxford, England : 1990). 2008:44(11):1541-51.

5. Hu Q, Luo Z, Xu T, Zhang JY, Zhu Y, Chen WX, et al. FOXA1: a promising prognostic marker in breast cancer. Asian Pacific J cancer prevention : APJCP. 2014;15(1):11-6.

6. Zhang G, Zhao Y, Liu Y, Kao LP, Wang X, Skerry B, et al. FOXA1 defines cancer cell specificity. Sci Adv. 2016;2(3):e1501473.

7. Augello MA, Hickey TE, Knudsen KE. FOXA1: master of steroid receptor function in cancer. EMBO J. 2011;30(19):3885-94.

8. Guiu S, Mollevi C, Charon-Barra C, Boissiere F, Crapez E, Chartron E, et al. Prognostic value of androgen receptor and FOXA1 co-expression in nonmetastatic triple negative breast cancer and correlation with other biomarkers. Br J Cancer. 2018;119(1):76-9.

9. Barbieri CE, Baca SC, Lawrence MS, Demichelis F, Blattner M, Theurillat JP, et al. Exome sequencing identifies recurrent SPOP, FOXA1 and MED12 mutations in prostate cancer. Nat Genet. 2012;44(6):685-9.

10. Grasso CS, Wu YM, Robinson DR, Cao X, Dhanasekaran SM, Khan AP, et al. The mutational landscape of lethal castration-resistant prostate cancer. Nature. 2012;487(7406):239-43.

11. Nakshatri H, Badve S. FOXA1 in breast cancer. Expert Rev Mol Med. 2009;11:e8.

12. Anzai E, Hirata K, Shibazaki M, Yamada C, Morii M, Honda T, et al. FOXA1 induces E-cadherin expression at the protein level via suppression of slug in epithelial breast Cancer cells. Biol Pharm Bull. 2017;40(9):1483-9.

13. Hisamatsu Y, Tokunaga E, Yamashita N, Akiyoshi S, Okada S, Nakashima Y, et al. Impact of GATA-3 and FOXA1 expression in patients with hormone receptor-positive/HER2-negative breast cancer. Breast cancer (Tokyo, Japan). 2015;22(5):520-8.

14. Ijichi N, Shigekawa T, Ikeda K, Horie-Inoue K, Shimizu C, Saji S, et al. Association of double-positive FOXA1 and FOXP1 immunoreactivities with favorable prognosis of tamoxifen-treated breast cancer patients. Hormones \& cancer. 2012;3(4):147-59.

15. Yamaguchi N, Nakayama Y, Yamaguchi N. Down-regulation of Forkhead box protein A1 (FOXA1) leads to cancer stem cell-like properties in tamoxifen-resistant breast cancer cells through induction of interleukin-6. J Biol Chem. 2017;292(20):8136-48.

16. Horimoto Y, Arakawa A, Harada-Shoji N, Sonoue H, Yoshida Y, Himuro T, et al. Low FOXA1 expression predicts good response to neo-adjuvant 
chemotherapy resulting in good outcomes for luminal HER2-negative breast cancer cases. Br J Cancer. 2015;112(2):345-51.

17. Nowak A, Grzegrzolka J, Paprocka M, Piotrowska A, Rys J, Matkowski R, et al. Nestin-positive microvessel density is an independent prognostic factor in breast cancer. Int J Oncol. 2017;51(2):668-76.

18. Parry S, Savage K, Marchio C, Reis-Filho JS. Nestin is expressed in basal-like and triple negative breast cancers. J Clin Pathol. 2008;61(9):1045-50.

19. Feng W, Liu S, Zhu R, Li B, Zhu Z, Yang J, et al. SOX10 induced nestin expression regulates cancer stem cell properties of TNBC cells. Biochem Biophys Res Commun. 2017;485(2):522-8.

20. Nowak A, Grzegrzolka J, Kmiecik A, Piotrowska A, Matkowski R, Dziegiel P. Role of nestin expression in angiogenesis and breast cancer progression. Int J Oncol. 2018;52(2):527-35.

21. Tampakis A, Tampaki EC, Trafalis D, Nonni A, Kontzoglou K, Patsouris E, et al. Nestin and CD146 expression in metaplastic breast cancer: stem-cell therapy in need? Lessons reported from a male patient. Eur Rev Med Pharmacol Sci. 2017;21(18):4137-40

22. Piras F, lonta MT, Lai S, Perra MT, Atzori F, Minerba L, et al. Nestin expression associates with poor prognosis and triple negative phenotype in locally advanced (T4) breast cancer. European J histochemistry: EJH. 2011;55(4):e39.

23. Kruger $K$, Wik E, Knutsvik $G$, Nalwoga $H$, Klingen TA, Arnes JB, et al. Expression of nestin associates with BRCA1 mutations, a basal-like phenotype and aggressive breast cancer. Sci Rep. 2017;7(1):1089.

24. Albergaria A, Paredes J, Sousa B, Milanezi F, Carneiro V, Bastos J, et al. Expression of FOXA1 and GATA-3 in breast cancer: the prognostic significance in hormone receptor-negative tumours. Breast cancer research: BCR. 2009;11(3):R40.

25. Mehta RJ, Jain RK, Leung S, Choo J, Nielsen T, Huntsman D, et al. FOXA1 is an independent prognostic marker for ER-positive breast cancer. Breast Cancer Res Treat. 2012;131(3):881-90.

26. Thorat MA, Marchio C, Morimiya A, Savage K, Nakshatri H, Reis-Filho JS, et al. Forkhead box A1 expression in breast cancer is associated with luminal subtype and good prognosis. J Clin Pathol. 2008;61(3):327-32.

27. Ademuyiwa FO, Thorat MA, Jain RK, Nakshatri H, Badve S. Expression of Forkhead-box protein $\mathrm{A} 1$, a marker of luminal a type breast cancer, parallels low Oncotype DX 21-gene recurrence scores. Modern pathology : an official journal of the United States and Canadian Academy of Pathology, Inc 2010;23(2):270-5

28. Kawase M, Toyama T, Takahashi S, Sato S, Yoshimoto N, Endo Y, et al. FOXA1 expression after neoadjuvant chemotherapy is a prognostic marke in estrogen receptor-positive breast cancer. Breast cancer (Tokyo, Japan). 2015;22(3):308-16

29. Robbins CM, Tembe WA, Baker A, Sinari S, Moses TY, Beckstrom-Sternberg S, et al. Copy number and targeted mutational analysis reveals novel somatic events in metastatic prostate tumors. Genome Res. 2011;21(1):47-55.

30. Schrijver W, Schuurman K, van Rossum A, Droog M, Jeronimo C, Salta S, et al. FOXA1 levels are decreased in pleural breast cancer metastases after adjuvant endocrine therapy, and this is associated with poor outcome. Mol Oncol. 2018;12(11):1884-94.

31. Asleh K, Won JR, Gao D, Voduc KD, Nielsen TO. Nestin expression in breast cancer: association with prognosis and subtype on 3641 cases with longterm follow-up. Breast Cancer Res Treat. 2018;168(1):107-15.

32. De Lara S, Parris TZ, Werner Ronnerman E, Helou K, Kovacs A. GATA3 as a putative marker of breast cancer metastasis-a retrospective immunohistochemical study. Breast J. 2018;24(2):184-8.

Ready to submit your research? Choose BMC and benefit from:

- fast, convenient online submission

- thorough peer review by experienced researchers in your field

- rapid publication on acceptance

- support for research data, including large and complex data types

- gold Open Access which fosters wider collaboration and increased citations

- maximum visibility for your research: over $100 \mathrm{M}$ website views per year

At BMC, research is always in progress.

Learn more biomedcentral.com/submissions 